Supporting Information

\title{
Versatile 5'-Functionalization of DNA Oligonucleotides on Solid Support: Amines, Azides, Thiols, and Thioethers via Phosphorus Chemistry
}

Gregory P. Miller and Eric T. Kool*

Department of Chemistry, Stanford University, Stanford CA 94305

\section{Table of Contents}

Table S1. Characterization data for 5' ${ }^{\prime}$-modified oligodeoxyribonucleotides...........2

Table S2: Procedure for conversion of 5'-OH oligodeoxyribonucleotides to 5'-SR on ABI 392 and 394 DNA synthesizers.........................4

Characterization: NMR spectra of 5'-modifications on the sequence dTTT..........5-20 
Table S1. Characterization data for 5'-modified oligodeoxyribonucleotides.

\begin{tabular}{|c|c|c|c|c|c|}
\hline Compound & $\begin{array}{l}\text { HPLC RT } \\
(\mathrm{min})\end{array}$ & $\begin{array}{l}\text { HPLC } \\
\text { method }^{1}\end{array}$ & $\begin{array}{l}\text { Mass expect } \\
\mathrm{m} / \mathrm{z}\end{array}$ & $\begin{array}{l}\text { Mass found } \\
\mathrm{m} / \mathrm{z}^{2}\end{array}$ & Peak \\
\hline Nap-S-TTT & 19.6 & $\mathrm{~B}$ & 991.2 & 991.2 & M-1 \\
\hline Nap-S-GTT & 17.5 & B & 1017.2 & 1016.1 & M-1 \\
\hline Nap-S-ATT & 18.6 & B & 1000.2 & 1000.2 & M-1 \\
\hline Nap-S-CTT & 17.7 & B & 976.2 & 976.2 & M-1 \\
\hline Me-S-TTT & 19.2 & A & 879.2 & 879.3 & M-1 \\
\hline Me-S-GTT & 16.2 & A & 904.2 & 904.1 & M-1 \\
\hline Me-S-ATT & 17.6 & A & 888.2 & 888.2 & M-1 \\
\hline Me-S-CTT & 16.4 & A & 864.2 & 864.1 & M-1 \\
\hline Pr-S-TTT & 21.8 & A & 907.2 & 907.1 & M-1 \\
\hline $\mathrm{C}_{12} \mathrm{H}_{26}-\mathrm{S}-\mathrm{TTT}$ & 29.8 & $\mathrm{C}$ & 1033.3 & 1033.3 & M-1 \\
\hline $\mathrm{Et}_{2} \mathrm{NCSS}-\mathrm{TTT}$ & 17.6 & $\mathrm{~B}$ & 980.2 & 980.1 & M-1 \\
\hline $\mathrm{Et}_{2} \mathrm{NCSS}-\mathrm{GTT}$ & 15.9 & $\mathrm{~B}$ & 1007.2 & 1006.9 & $\mathrm{M}+1$ \\
\hline $\mathrm{Et}_{2} \mathrm{NCSS}-\mathrm{ATT}$ & 17.0 & B & 989.2 & 989.2 & M-1 \\
\hline $\mathrm{Et}_{2} \mathrm{NCSS}-\mathrm{CTT}$ & 16.2 & B & 967.2 & 966.9 & $\mathrm{M}+1$ \\
\hline $\mathrm{Et}_{2} \mathrm{NCSS}-\mathrm{T} 7 \mathrm{~N}^{3}$ & 13.3 & $\mathrm{~B}$ & 1107.2 & 1107.1 & $(\mathrm{M}-2) / 2$ \\
\hline $\mathrm{Et}_{2} \mathrm{NCSS}-\mathrm{G} 7 \mathrm{~N}^{3}$ & 12.5 & B & 1144.7 & 1144.7 & $(\mathrm{M}-2) / 2$ \\
\hline $\mathrm{Et}_{2} \mathrm{NCSS}-\mathrm{A} 7 \mathrm{~N}^{3}$ & 13.0 & B & 1120.7 & 1120.6 & $(\mathrm{M}-2) / 2$ \\
\hline $\mathrm{Et}_{2} \mathrm{NCSS}-\mathrm{C} 7 \mathrm{~N}^{3}$ & 12.6 & B & 1084.7 & 1085.1 & $(\mathrm{M}-2) / 2$ \\
\hline HS-TTT & 13.4 & $\mathrm{~B}$ & 865.2 & 865.3 & M-1 \\
\hline HS-GTT & 12.0 & B & 892.2 & 891.9 & $\mathrm{M}+1$ \\
\hline HS-ATT & 12.9 & $\mathrm{~B}$ & 874.2 & 874.3 & M-1 \\
\hline HS-CTT & 11.3 & $\mathrm{~B}$ & 850.2 & 850.3 & M-1 \\
\hline HS-T7N ${ }^{3}$ & 11.2 & $\mathrm{~B}$ & 1050.7 & 1049.6 & $(\mathrm{M}-2) / 2$ \\
\hline HS-G7N ${ }^{3}$ & 10.4 & B & 1087.2 & 1087.1 & $(\mathrm{M}-2) / 2$ \\
\hline $\mathrm{HS}-\mathrm{A} 7 \mathrm{~N}^{3}$ & 10.9 & B & 1063.2 & 1063.2 & $(\mathrm{M}-2) / 2$ \\
\hline $\mathrm{HS}-\mathrm{C} 7 \mathrm{~N}^{3}$ & 10.3 & B & 1027.2 & 1027.7 & $(\mathrm{M}-2) / 2$ \\
\hline $\mathrm{N}_{3}-\mathrm{TTT}$ & 19.3 & A & 874.2 & 874.1 & M-1 \\
\hline $\mathrm{N}_{3}-\mathrm{GTT}$ & 16.5 & A & 899.2 & 899.3 & M-1 \\
\hline $\mathrm{N}_{3}-\mathrm{ATT}$ & 17.7 & A & 883.2 & 883.1 & M-1 \\
\hline $\mathrm{N}_{3}-\mathrm{CTT}$ & 16.5 & A & 859.2 & 859.1 & M-1 \\
\hline $\mathrm{H}_{2} \mathrm{~N}-\mathrm{TTT}$ & 12.9 & A & 848.2 & 848.1 & M-1 \\
\hline $\mathrm{H}_{2} \mathrm{~N}-\mathrm{GTT}$ & 11.8 & A & 873.2 & 873.2 & M-1 \\
\hline $\mathrm{H}_{2} \mathrm{~N}-\mathrm{ATT}$ & 12.6 & A & 857.2 & 857.2 & M-1 \\
\hline $\mathrm{H}_{2} \mathrm{~N}-\mathrm{CTT}$ & 11.2 & A & 835.2 & 835.0 & $\mathrm{M}+1$ \\
\hline $\mathrm{H}_{2} \mathrm{~N}-\mathrm{T}_{5}$ & 14.6 & A & 1456.3 & 1456.1 & M-1 \\
\hline $\mathrm{H}_{2} \mathrm{~N}-\mathrm{G}_{5}$ & 11.2 & A & 1581.3 & 1581.6 & M-1 \\
\hline $\mathrm{H}_{2} \mathrm{~N}-\mathrm{A}_{5}$ & 12.6 & A & 1501.3 & 1501.2 & M-1 \\
\hline $\mathrm{H}_{2} \mathrm{~N}-\mathrm{C}_{5}$ & 11.1 & A & 1381.3 & 1381.3 & M-1 \\
\hline $\mathrm{N}_{3}-\mathrm{T}_{7 \mathrm{~N}^{3}}$ & 16.9 & A & 2312.4 & 2312.0 & $\mathrm{M}+\left(\mathrm{NEt}_{3}\right) 2$ \\
\hline $\mathrm{N}_{3}-\mathrm{G} 7 \mathrm{~N}^{3}$ & 15.2 & A & 1091.7 & 1091.7 & $(\mathrm{M}-2) / 2$ \\
\hline$N_{3}-A 7 N^{3}$ & 15.9 & A & 1067.7 & 1067.7 & $(\mathrm{M}-2) / 2$ \\
\hline $\mathrm{N}_{3}-\mathrm{C} 7 \mathrm{~N}^{3}$ & 16.1 & A & 1031.7 & 1031.7 & $(\mathrm{M}-2) / 2$ \\
\hline
\end{tabular}




\begin{tabular}{llllll}
\hline p- ${ }_{2} \mathrm{~N}-\mathrm{Ph}-\mathrm{S}-\mathrm{TTT}$ & 15.4 & $\mathrm{~B}$ & 956.2 & 956.3 & $\mathrm{M}-1$ \\
$\mathrm{o}-\mathrm{H}_{2} \mathrm{~N}-\mathrm{Ph}-\mathrm{S}-\mathrm{TTT}$ & 16.1 & $\mathrm{~B}$ & 956.2 & 956.4 & $\mathrm{M}-1$ \\
$\mathrm{~m}-\mathrm{HS}-\mathrm{Ph}-\mathrm{S}-\mathrm{TTT}$ & 17.8 & $\mathrm{~B}$ & 973.2 & 973.4 & $\mathrm{M}-1$
\end{tabular}

\footnotetext{
${ }^{1}$ HPLC method A: 0-20\% $\mathrm{CH}_{3} \mathrm{CN}$ in 50mM triethylammonium acetate buffer (TEAA) over 20 min, then 5 $\min 100 \% \mathrm{CH}_{3} \mathrm{CN}$, then 5 min $100 \%$ TEAA. HPLC method B: $0-36 \% \mathrm{CH}_{3} \mathrm{CN}$ in 50mM TEAA over 20 min, then 5 min $100 \% \mathrm{CH}_{3} \mathrm{CN}$, then 5 min $100 \%$ TEAA. HPLC method C: $0-54 \% \mathrm{CH}_{3} \mathrm{CN}$ in $50 \mathrm{mM}$ TEAA over $30 \mathrm{~min}$, then $5 \mathrm{~min} 100 \% \mathrm{CH}_{3} \mathrm{CN}$, then 5 min 100\% TEAA.

${ }^{2}$ All samples were analyzed on a ThermoFinnigan quadrupole ion trap LC-MS using positive and negative electrospray ionization.

${ }^{3}$ T7N=5'-dTCTGTAT-3'; G7N=5'-dGCGAGTG-3'; A7N=5'-dACAGATA-3'; C7N=5'-dCACGCTC-3'
} 
Table S2. Procedure for conversion of 5'-OH oligodeoxyribonucleotides to 5'-SR on ABI 392 and 394 DNA synthesizers.

\begin{tabular}{|c|c|c|}
\hline Step & Function & Time (sec) \\
\hline 1 & Begin & \\
\hline 2 & Prep DMF & 15 \\
\hline 3 & DMF to waste & 4 \\
\hline 4 & DMF to column & 15 \\
\hline 5 & Reverse flush & 10 \\
\hline 6 & Block flush & 4 \\
\hline 7 & Phos prep & 3 \\
\hline 8 & Iodo to column & 20 \\
\hline 9 & DMF to waste & 3 \\
\hline 10 & Block flush & 4 \\
\hline 11 & Wait & 300 \\
\hline 12 & Reverse flush & 10 \\
\hline 13 & Iodo to column & 20 \\
\hline 14 & DMF to waste & 3 \\
\hline 15 & Block flush & 4 \\
\hline 16 & Wait & 300 \\
\hline 17 & DMF to column & 15 \\
\hline 18 & Flush to waste & 10 \\
\hline 19 & DMF to column & 20 \\
\hline 20 & Flush to waste & 10 \\
\hline 21 & DMF to column & 20 \\
\hline 22 & \begin{tabular}{|l|} 
Reverse flush \\
\end{tabular} & 10 \\
\hline 23 & Block flush & 4 \\
\hline 24 & Phos prep & 3 \\
\hline 25 & Thiol to column & 20 \\
\hline 26 & DMF to waste & 4 \\
\hline 27 & Block flush & 3 \\
\hline 28 & Wait & 120 \\
\hline 29 & DMF to column & 15 \\
\hline 30 & Flush to waste & 10 \\
\hline 31 & DMF to column & 20 \\
\hline 32 & Flush to waste & 10 \\
\hline 33 & DMF to column & 20 \\
\hline 34 & \begin{tabular}{|l|} 
Reverse flush \\
\end{tabular} & 10 \\
\hline 35 & Block flush & 4 \\
\hline 36 & $\mathrm{CH}_{2} \mathrm{Cl}_{2}$ to waste & 3 \\
\hline 37 & $\mathrm{CH}_{2} \mathrm{Cl}_{2}$ to column & 15 \\
\hline 38 & Reverse flush & 10 \\
\hline 39 & Block flush & 4 \\
\hline 40 & End & \\
\hline
\end{tabular}




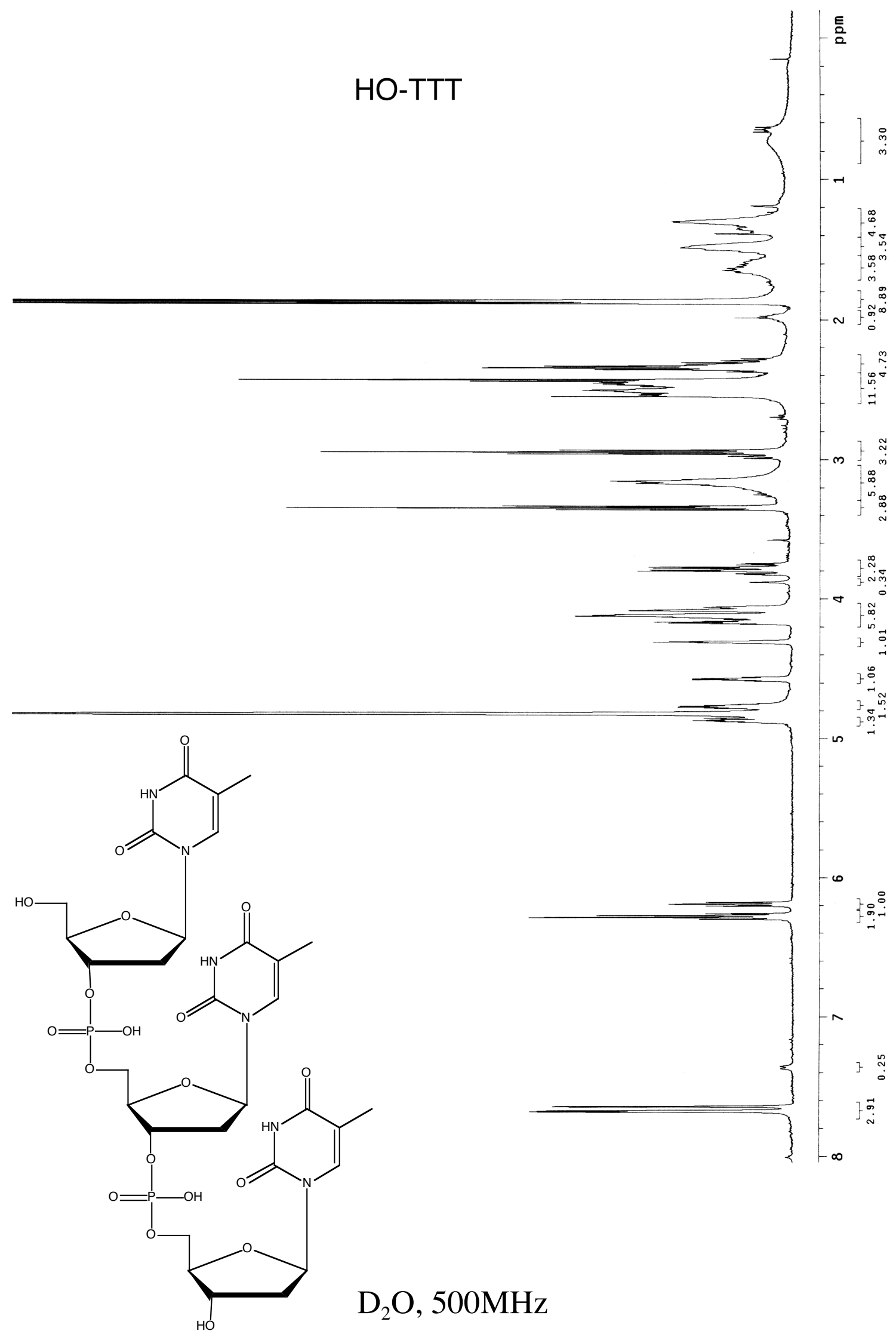




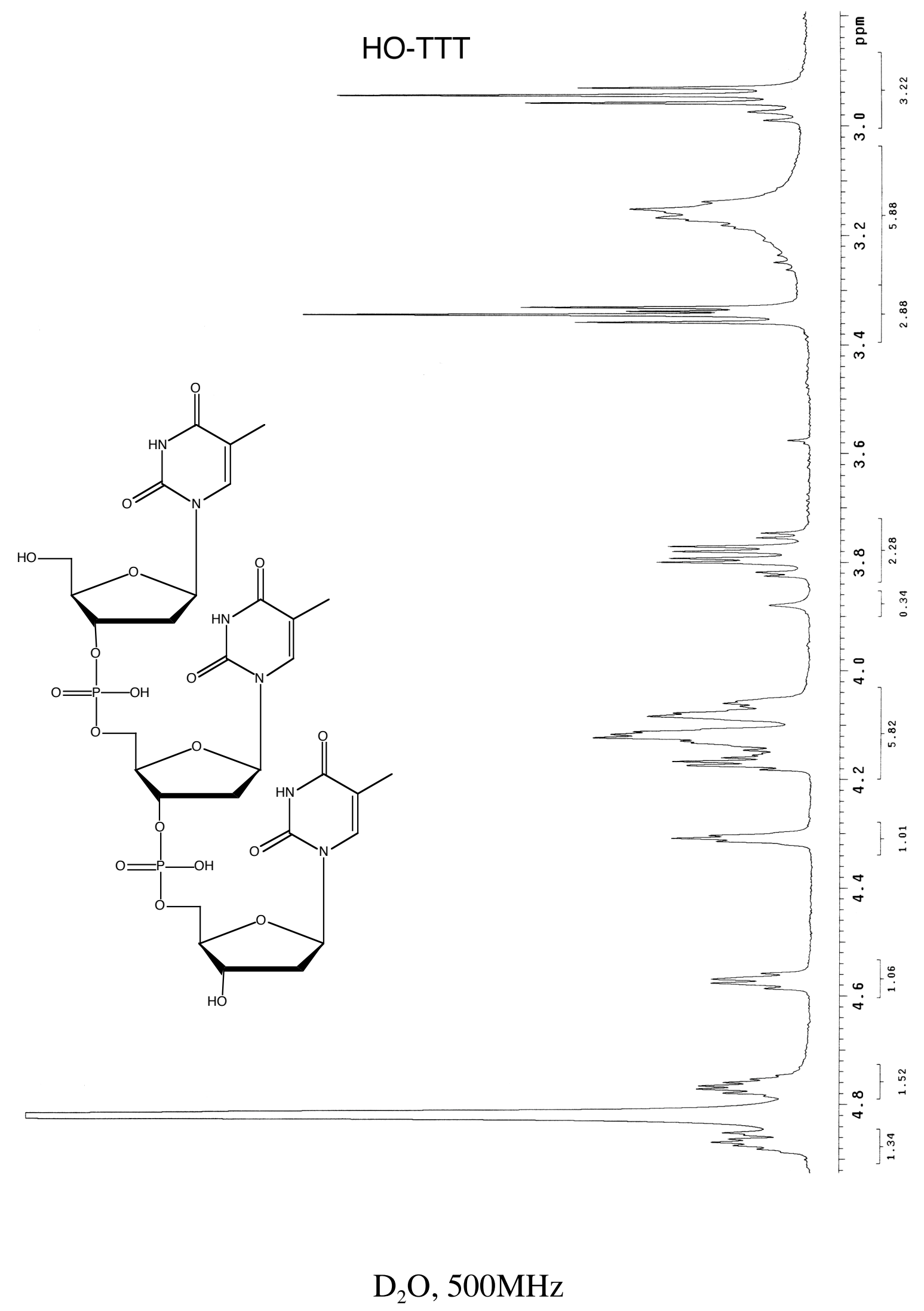




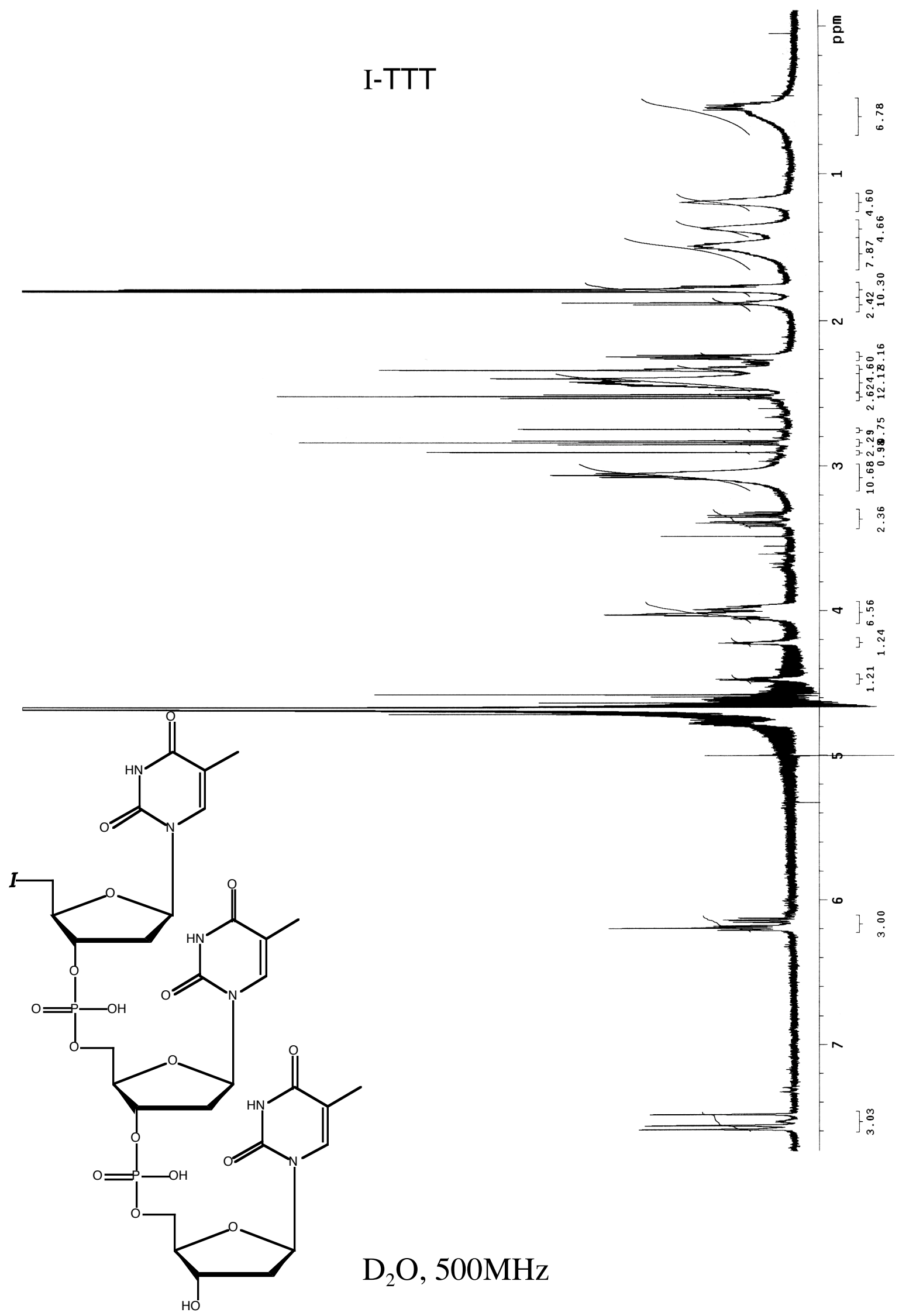




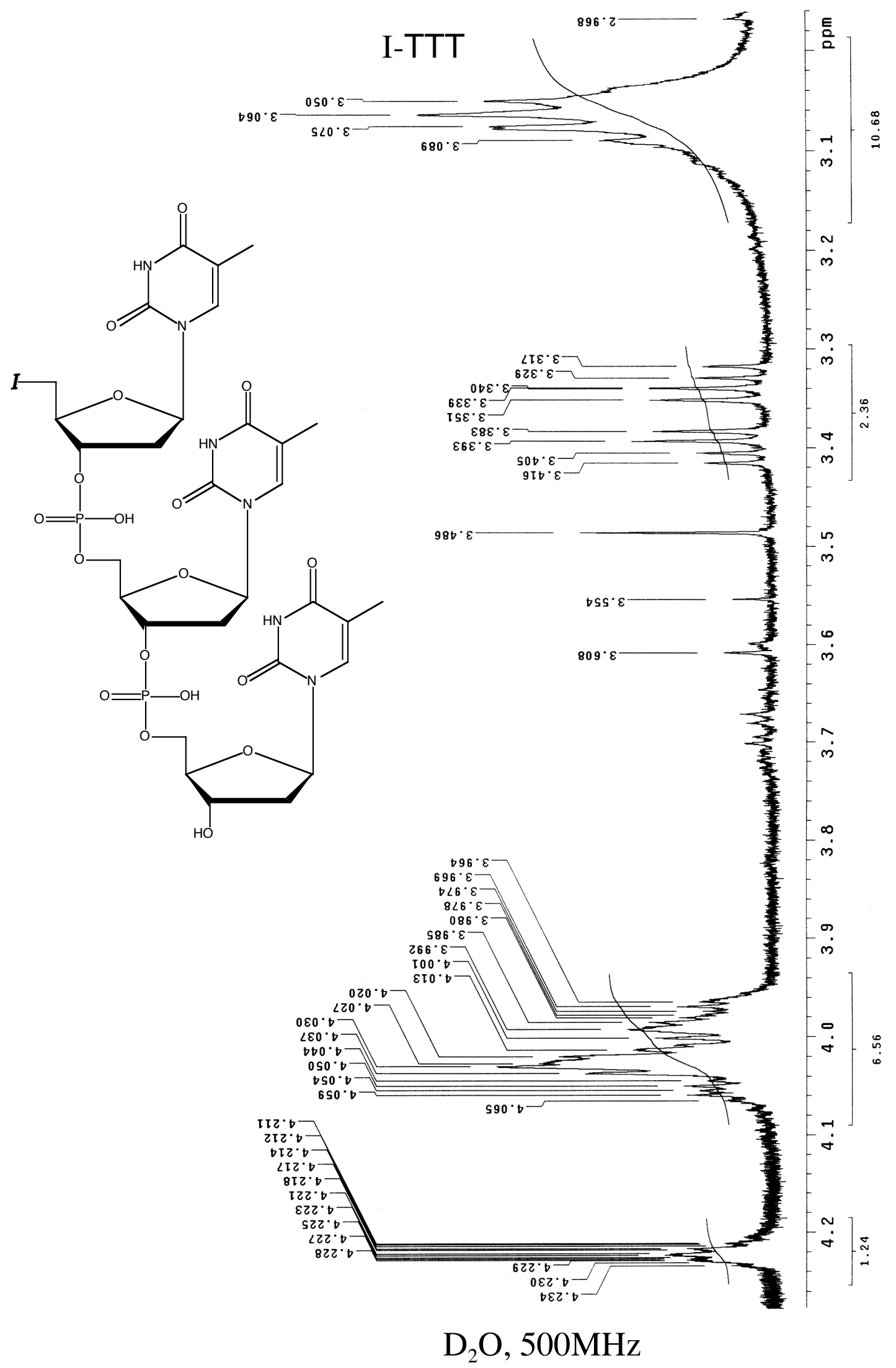




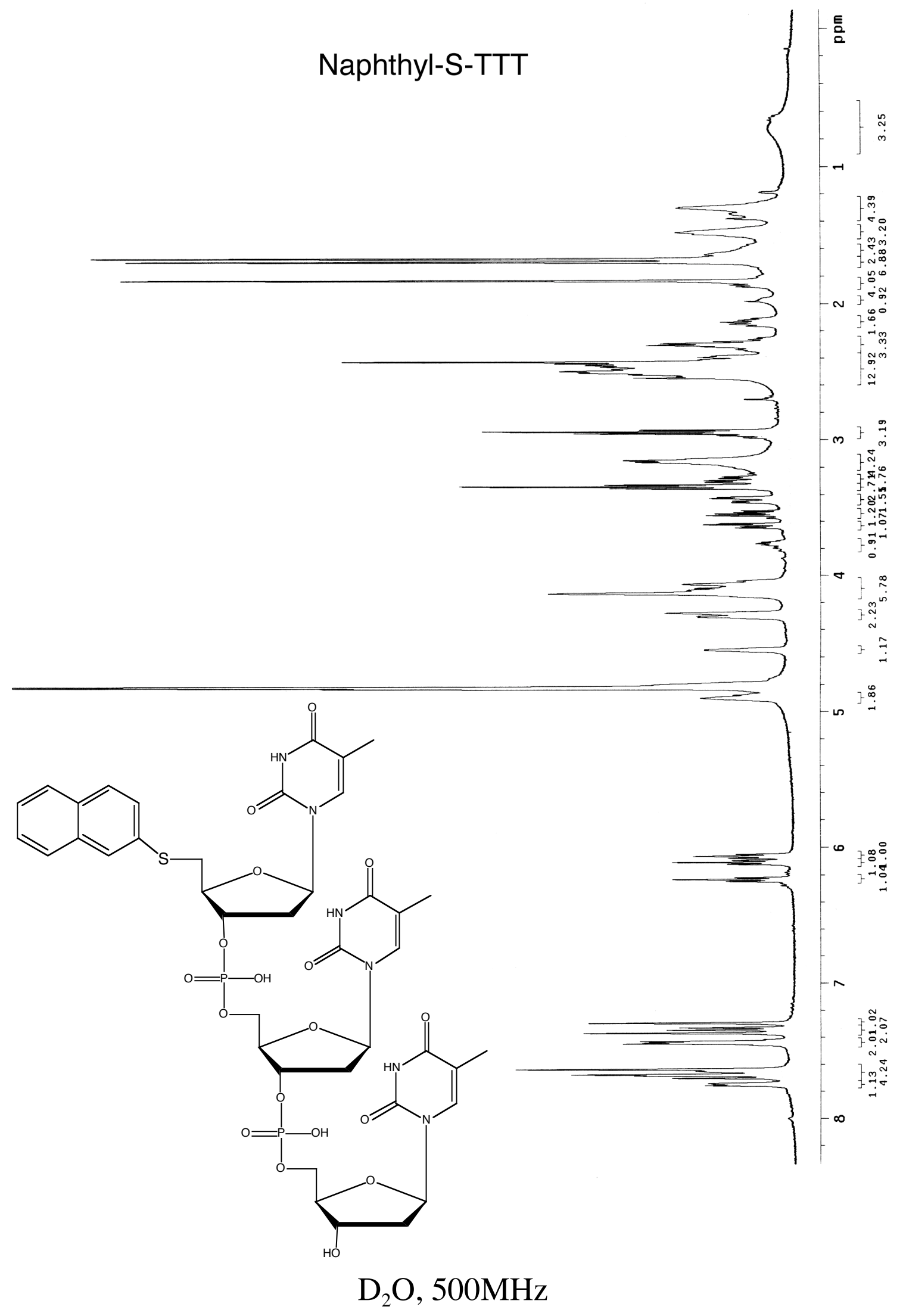




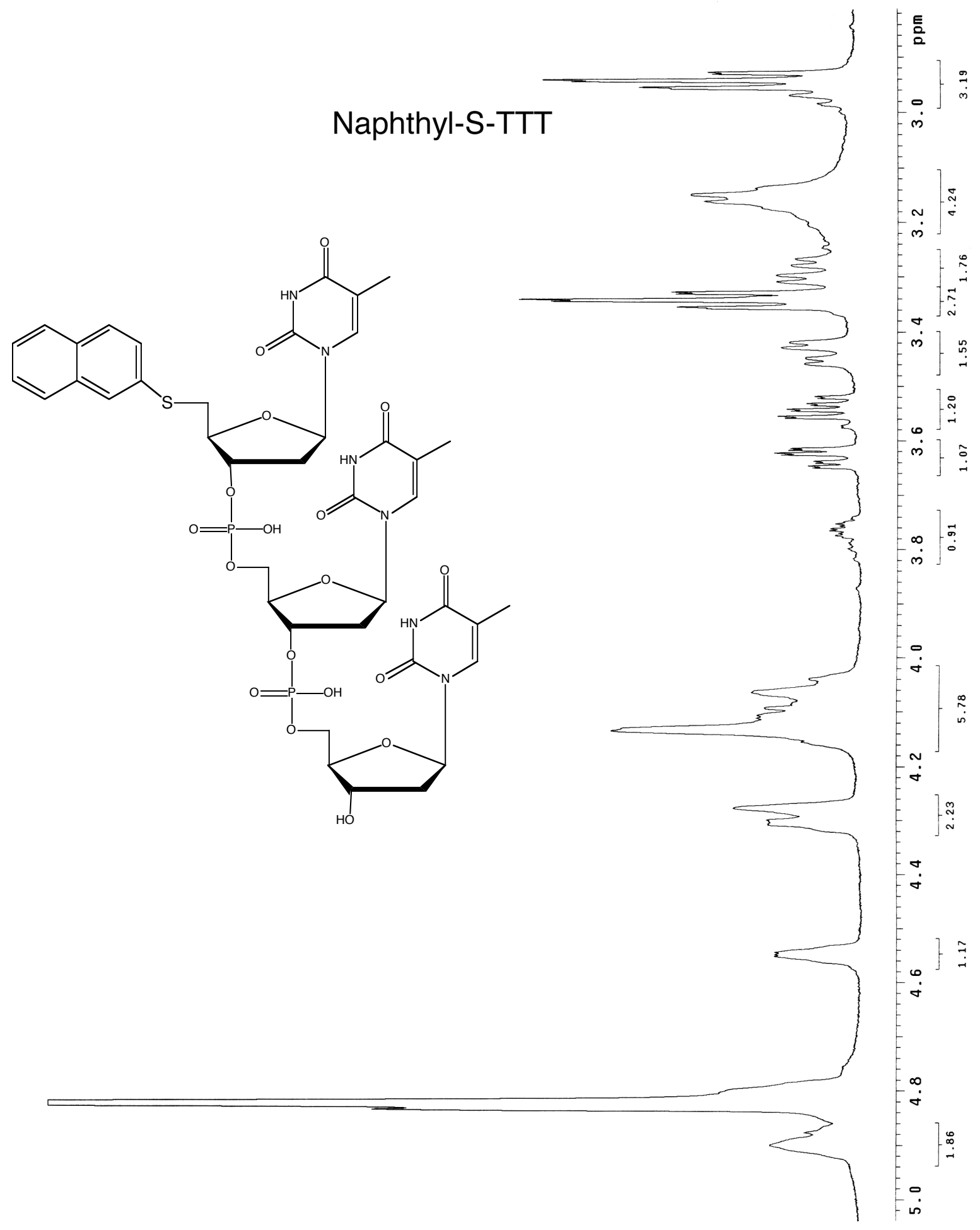

$\mathrm{D}_{2} \mathrm{O}, 500 \mathrm{MHz}$ 


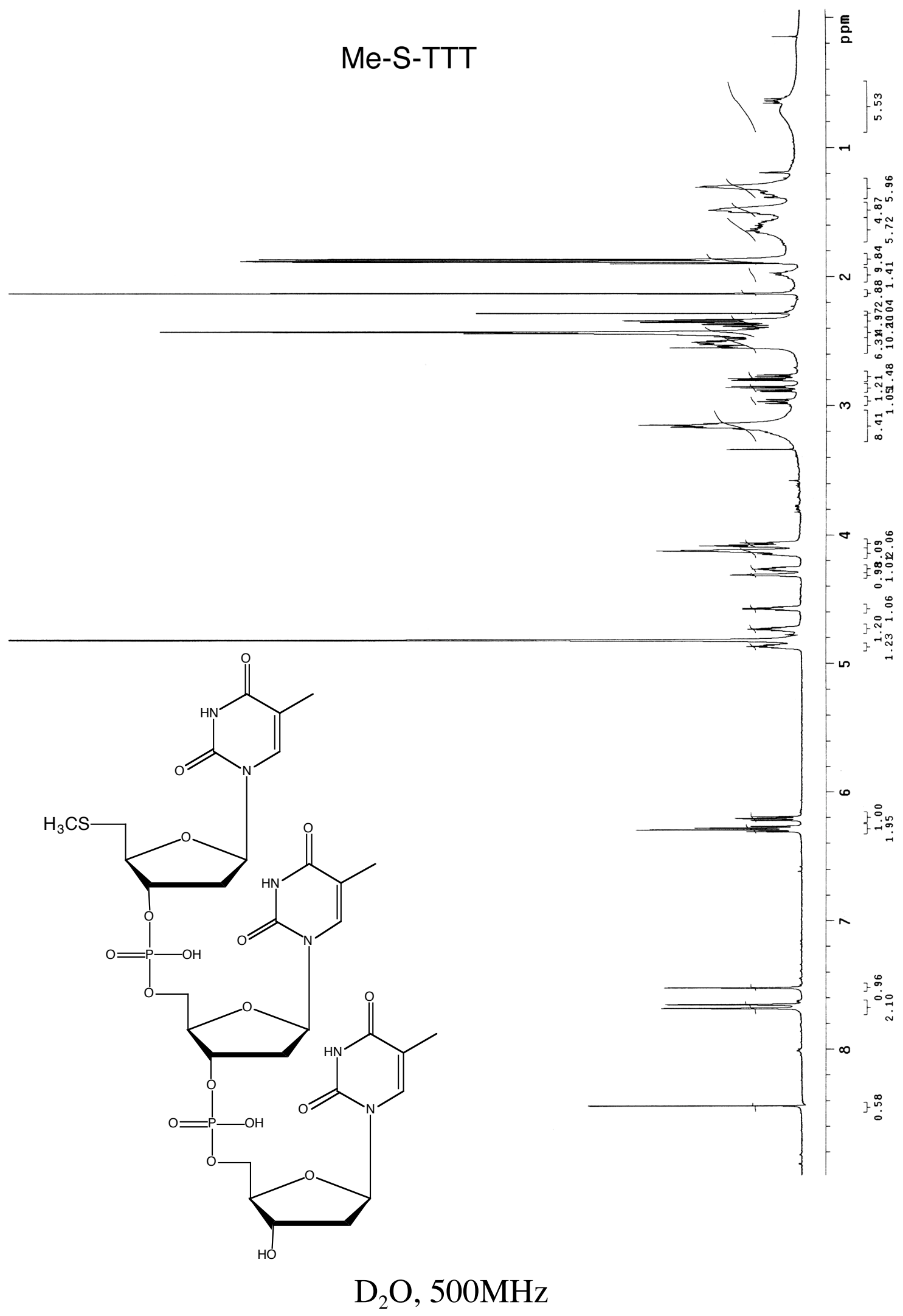




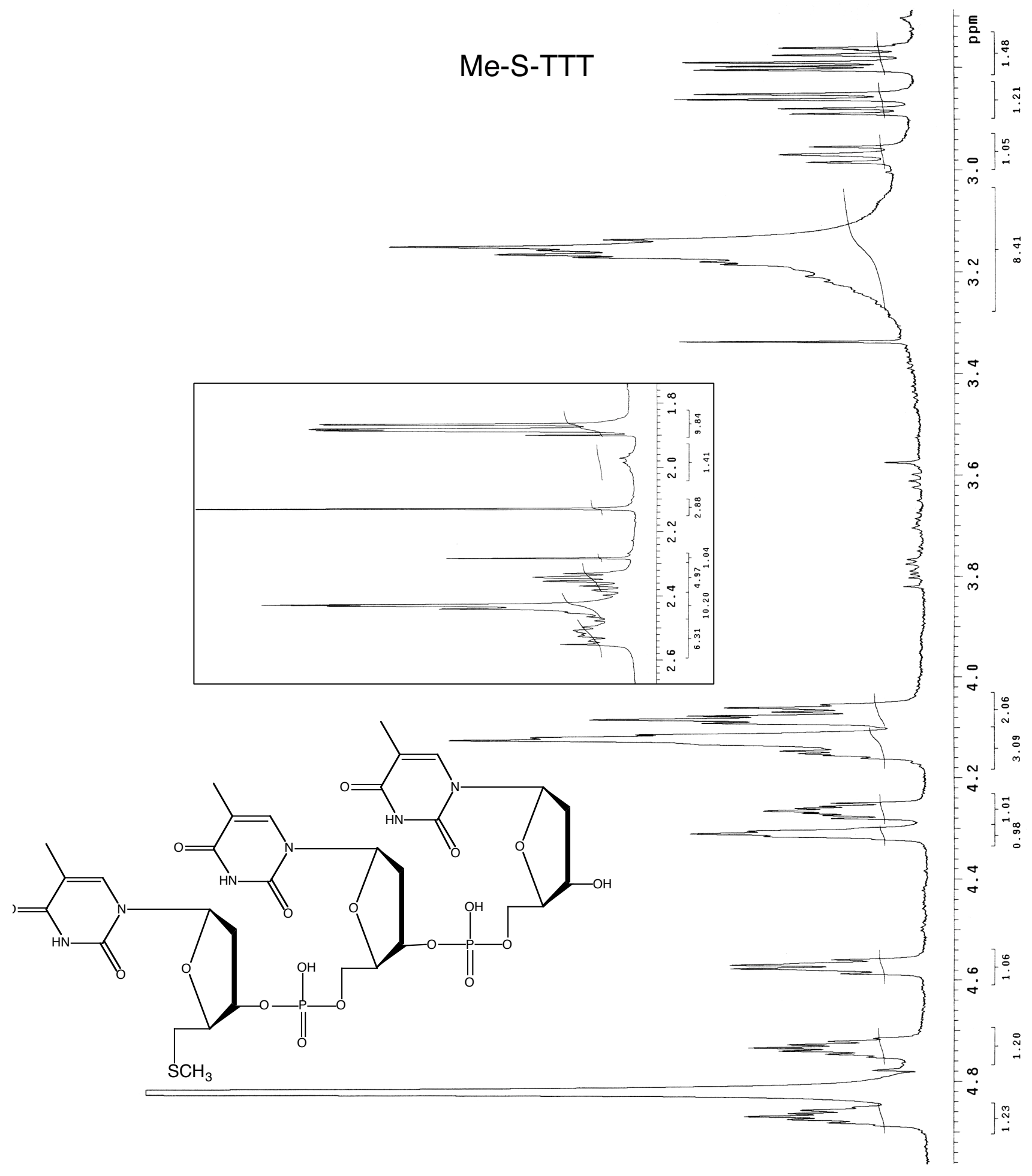

$\mathrm{D}_{2} \mathrm{O}, 500 \mathrm{MHz}$ 


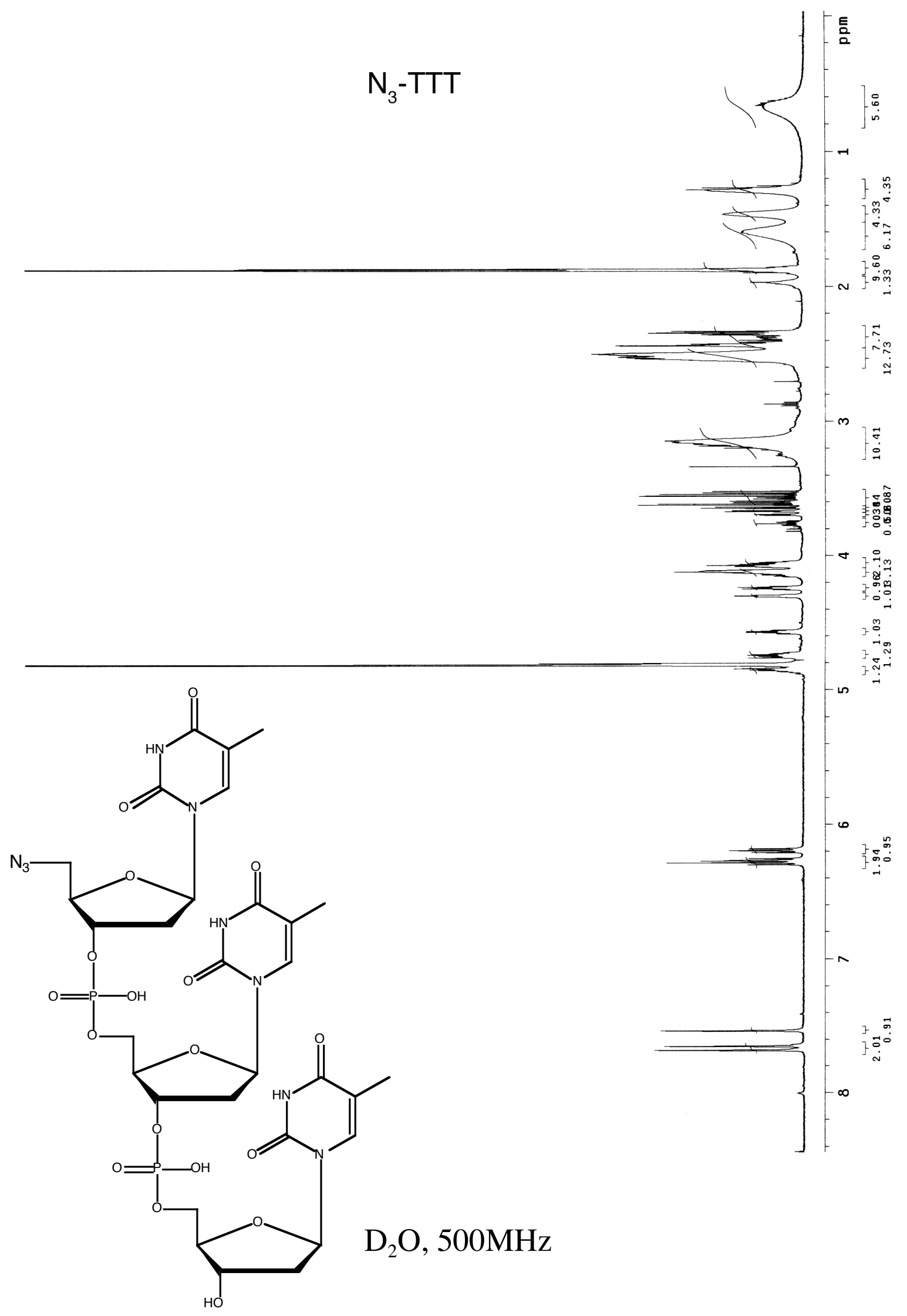




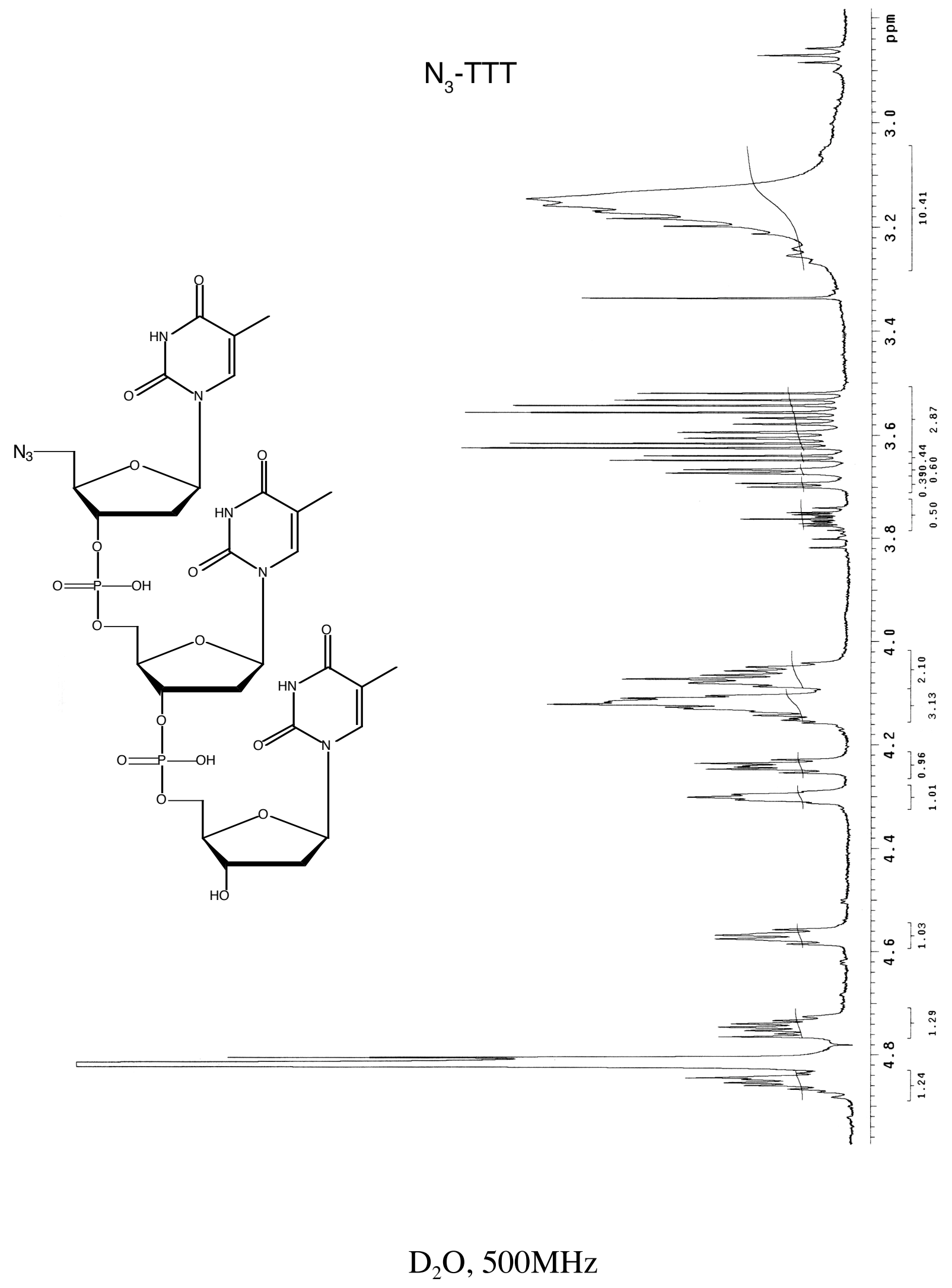




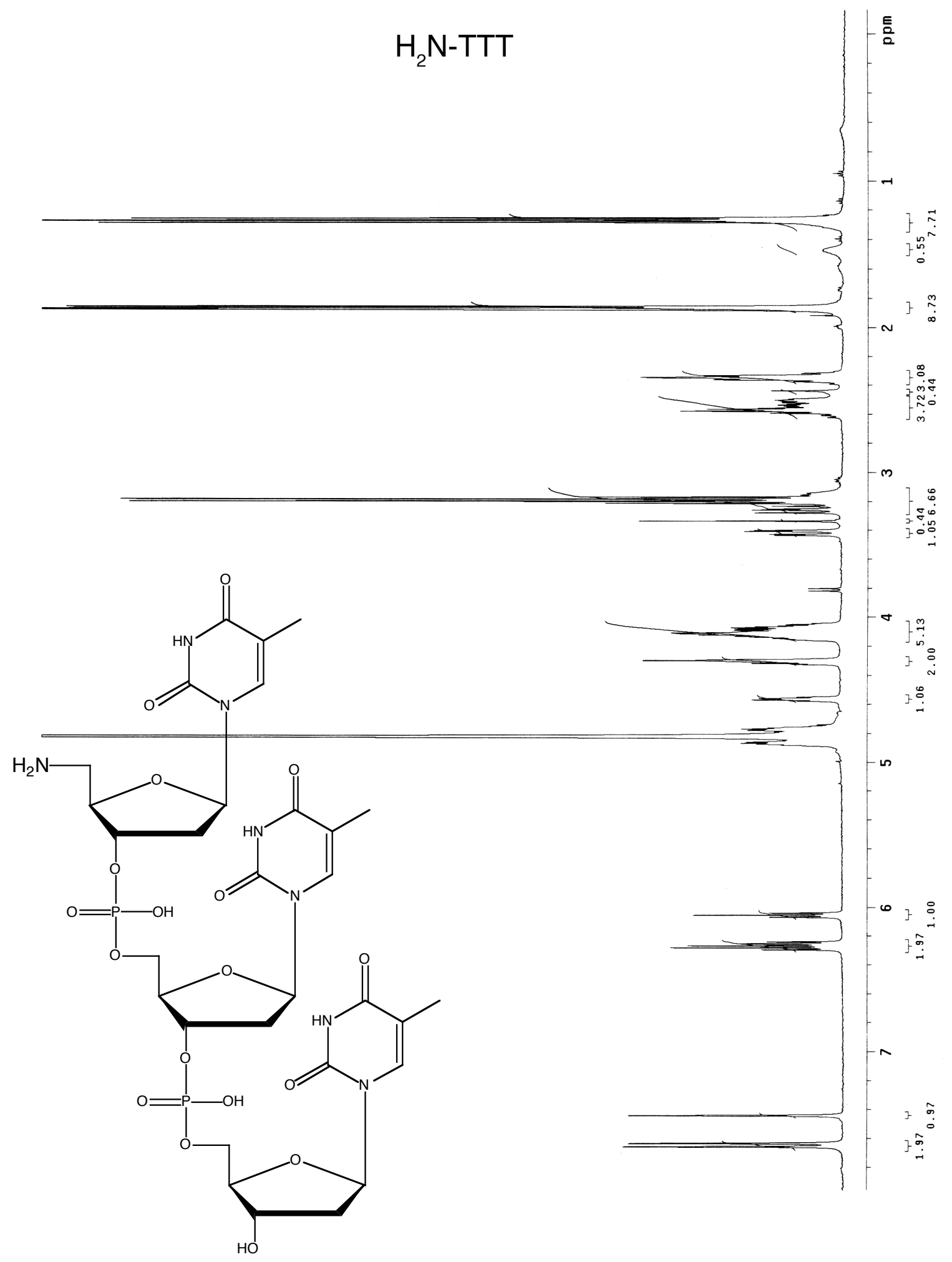

$\mathrm{D}_{2} \mathrm{O}, 500 \mathrm{MHz}$ 


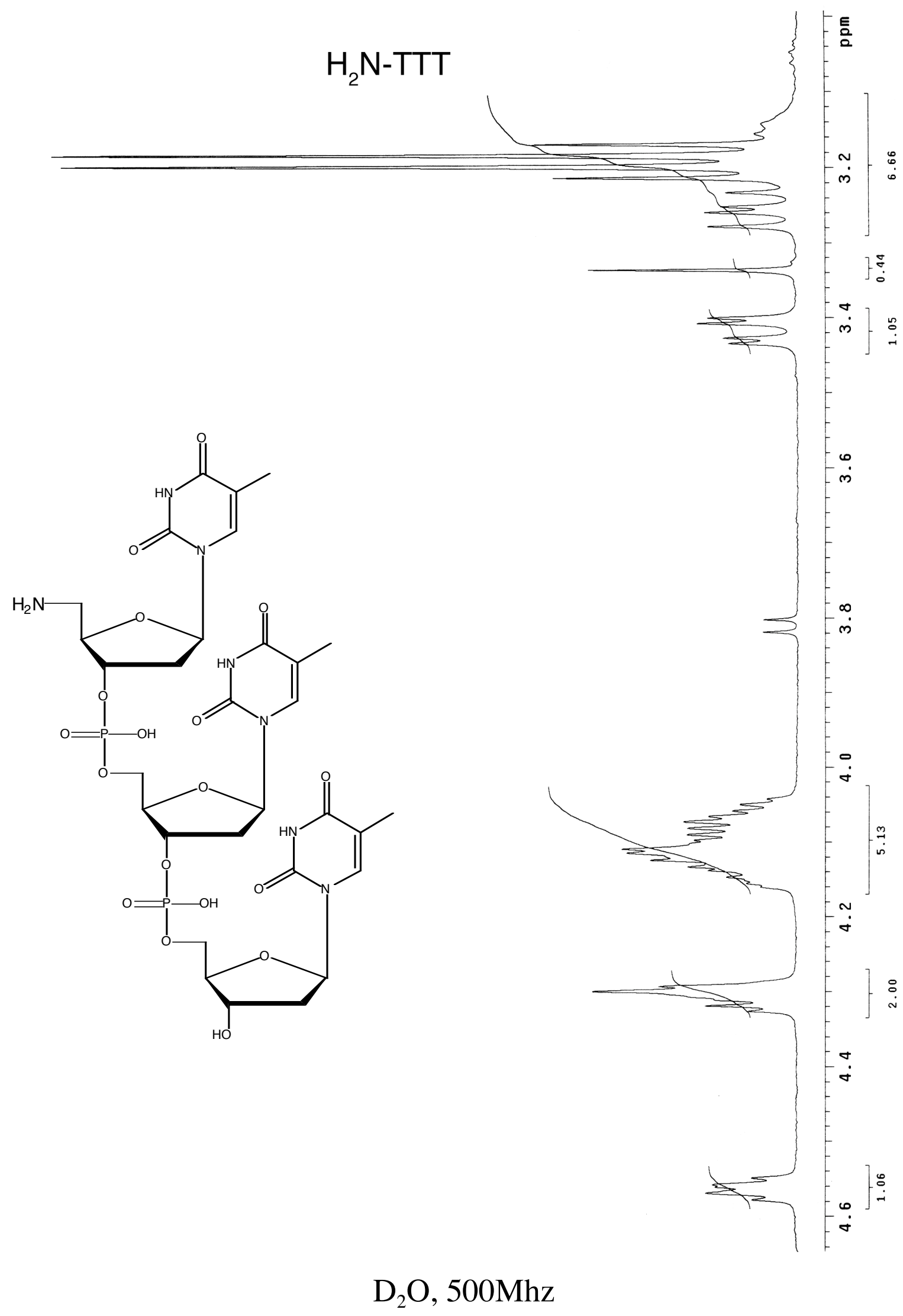




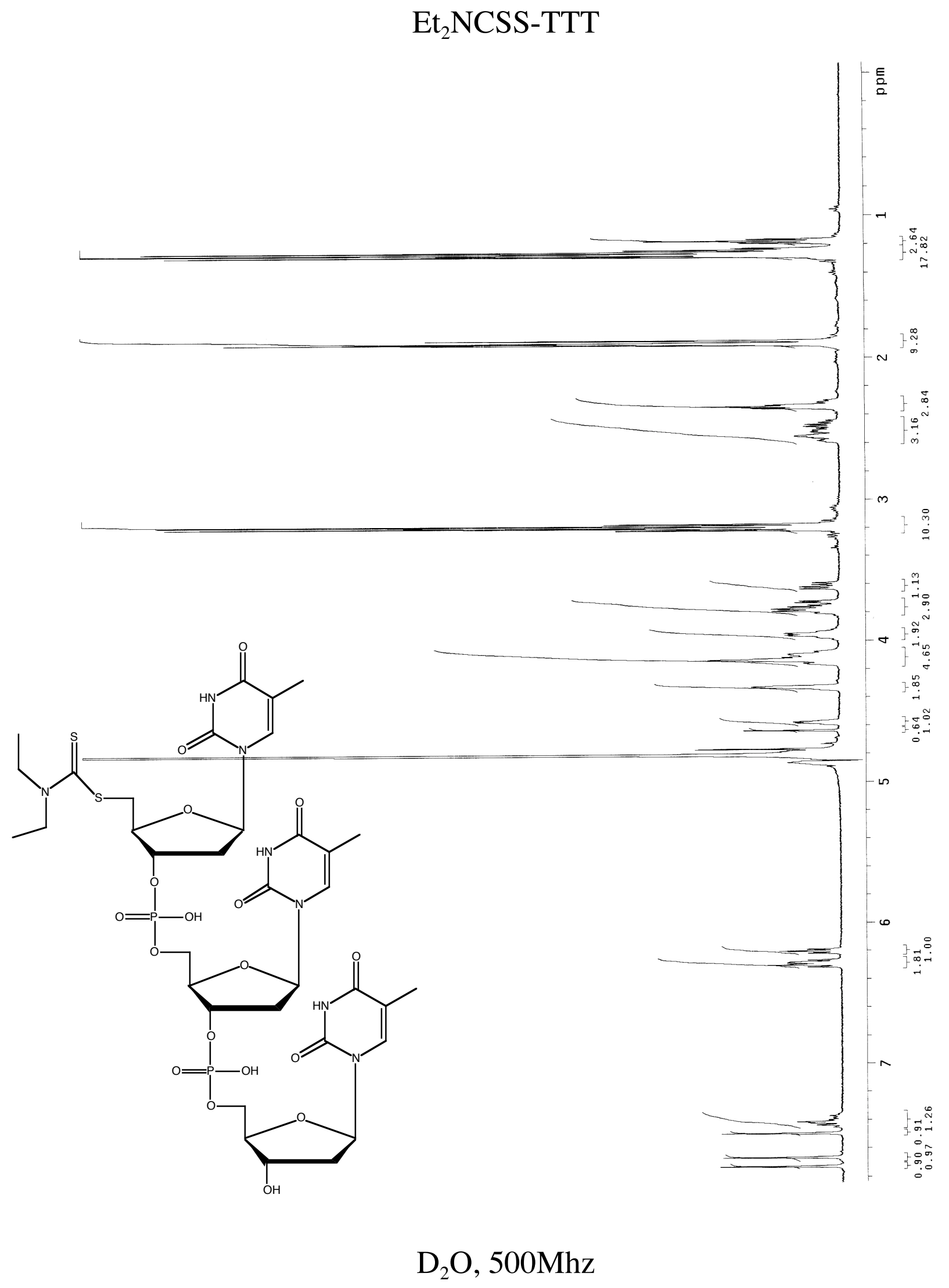




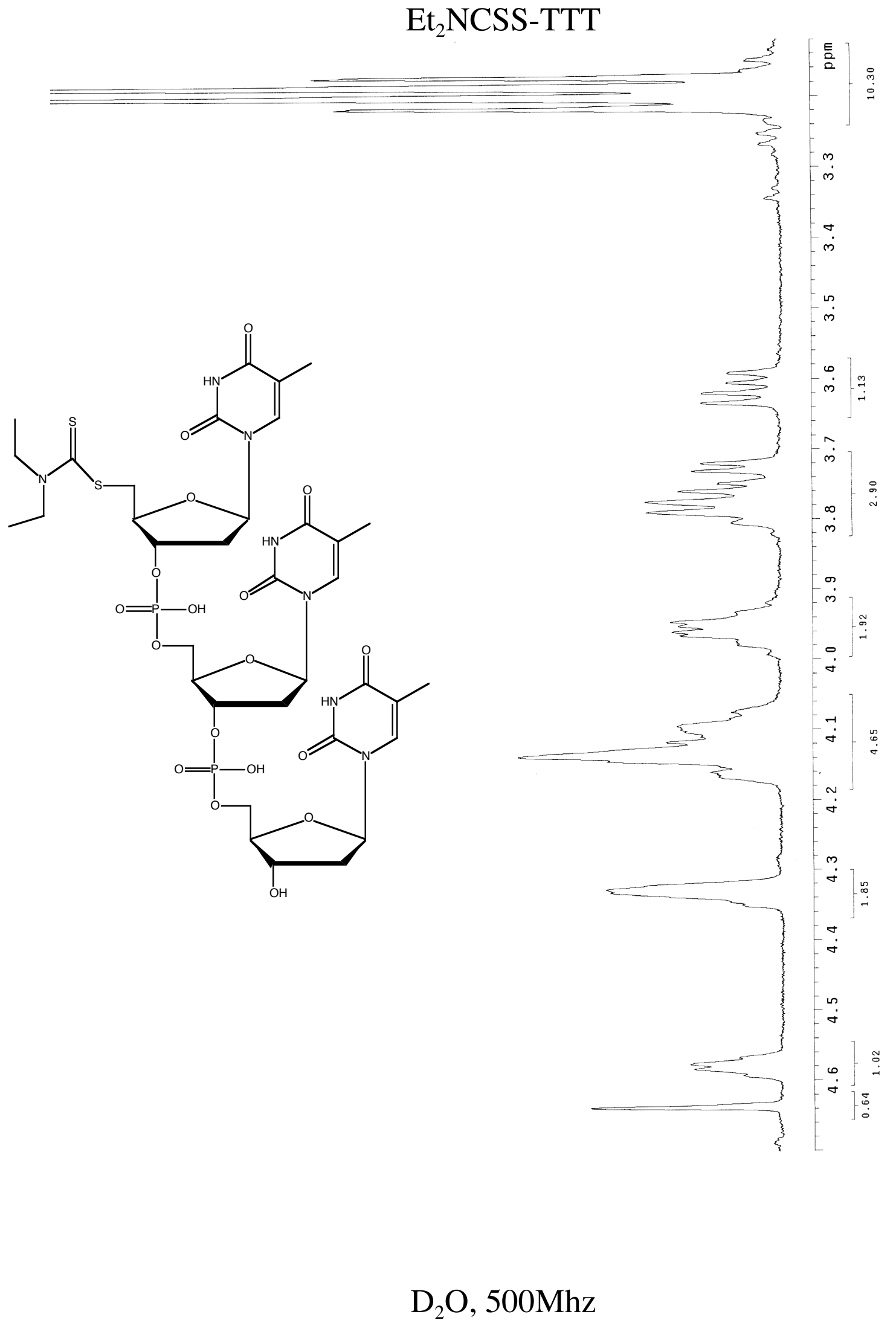




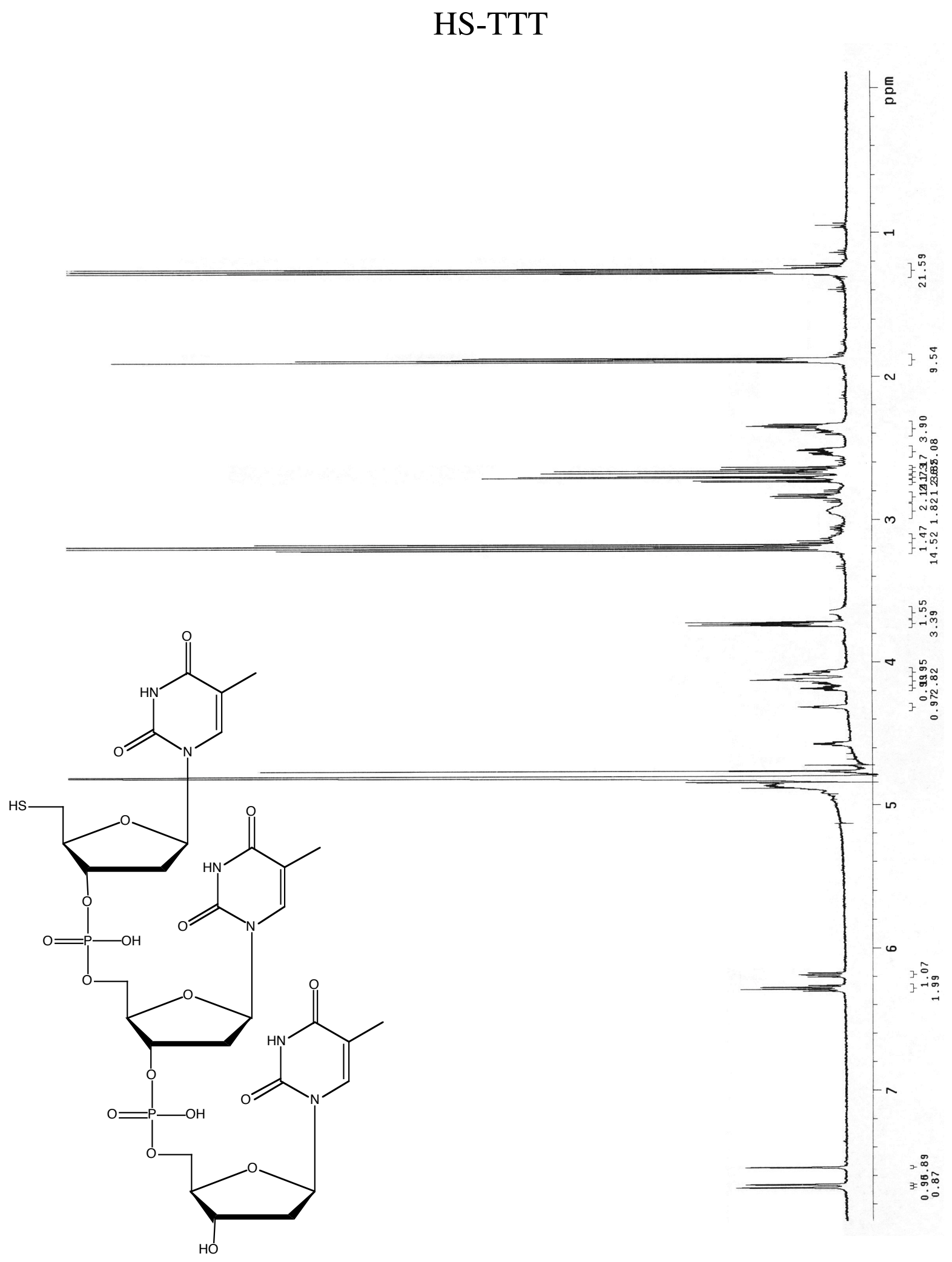

$\mathrm{D}_{2} \mathrm{O}, 500 \mathrm{Mhz}$ 


\section{HS-TTT}

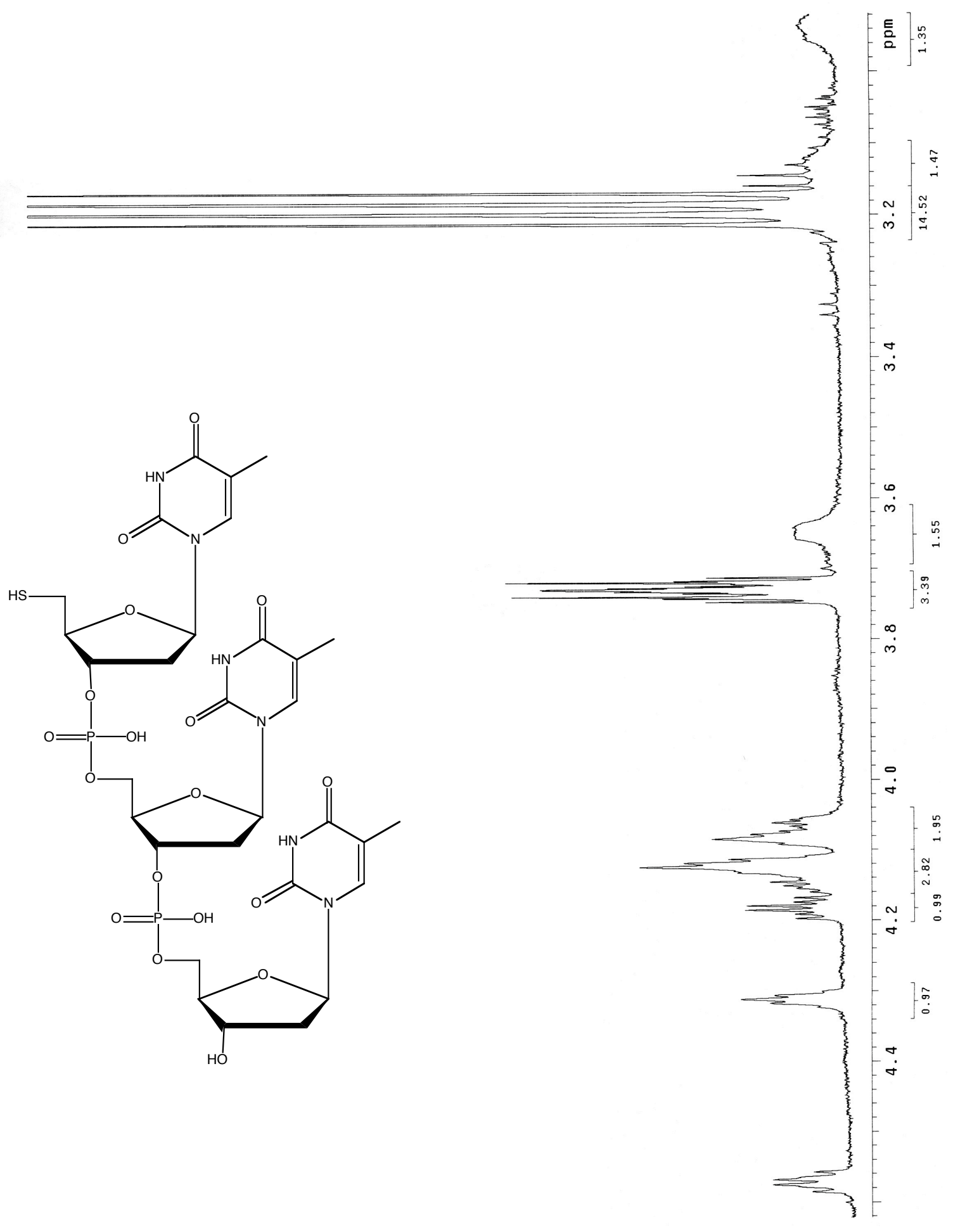

$\mathrm{D}_{2} \mathrm{O}, 500 \mathrm{Mhz}$ 